\title{
The long non-coding RNA HOTAIR is upregulated in endometrial carcinoma and correlates with poor prognosis
}

\author{
XIAOYING HE $^{1 *}$, WEI BAO ${ }^{*}$, XIAOCUI LI ${ }^{1},{\text { ZHENG } \mathrm{CHEN}^{2}, \text { QI CHE}^{2}, \text { HUIHUI WANG }^{2} \text { and XIAO-PING WAN }}^{2}$ \\ ${ }^{1}$ International Peace Maternity and Child Health Hospital Affiliated to Shanghai Jiao Tong University \\ School of Medicine, Shanghai 200030; ${ }^{2}$ Department of Obstetrics and Gynecology, Shanghai First People's Hospital \\ Affiliated to Shanghai Jiao Tong University School of Medicine, Shanghai 201620, P.R. China
}

Received August 13, 2013; Accepted November 15, 2013

DOI: $10.3892 /$ ijmm.2013.1570

\begin{abstract}
Long non-coding RNAs (lncRNAs) are emerging as key molecules in human cancer. Homeobox (HOX) transcript antisense intergenic RNA (HOTAIR), a long non-coding RNA (lncRNA), is associated with a variety of human cancers, such as breast, liver and lung cancer. However, whether HOTAIR can function as a molecular marker in endometrial carcinoma (EC) remains unknown. In the present study, the expression of HOTAIR in $66 \mathrm{EC}$ tissues from patients with $\mathrm{EC}$ and 30 normal tissues from healthy age-matched control subjects was determined using quantitative reverse transcription PCR. Furthermore, using in situ hybridization, we measured HOTAIR expression in 129 formalin-fixed paraffin-embedded (FFPE) tissue sections, which included 96 tissues that matched the frozen cases, 21 other EC tissues and 12 atypical hyperplasia tissues. Correlations between HOTAIR expression and the clinicopathological characteristics of patients were analyzed. Our results revealed that HOTAIR expression in the EC tissues was significantly upregulated compared with normal tissues $(p<0.001)$. In addition, we observed a significant association between HOTAIR expression and the EC grade $(\mathrm{p}<0.05)$ and lymph node metastasis $(\mathrm{p}<0.05)$. Moreover, in the FFPE tissues, but not the frozen tissues, we found that a higher HOTAIR expression also correlated with the depth of myometrial invasion $(\mathrm{p}=0.019)$ and lymphovascular space invasion $(\mathrm{p}=0.015)$. More importantly, patients with a higher HOTAIR expression showed significantly poorer overall survival than those with lower HOTAIR expression $(\mathrm{p}<0.05)$. In conclusion, our results
\end{abstract}

Correspondence to: Professor Xiao-Ping Wan, Department of Obstetrics and Gynecology, Shanghai First People's Hospital Affiliated to Shanghai Jiao Tong University School of Medicine, 650 New Songjiang Road, Shanghai 201620, P.R. China

E-mail:wanxp@sjtu.edu.cn

*Contributed equally

Key words: homeobox (HOX) transcript antisense intergenic RNA, long non-coding RNA, endometrial carcinoma, prognosis suggest that a high expression of HOTAIR is involved in the progression of cancer and may be a novel biomarker of poor prognosis in patients with EC.

\section{Introduction}

Endometrial carcinoma (EC) is one of the most common malignancies of the female reproductive system in Western countries. In 2013, an estimated 49,500 new cases and 8,200 deaths due to EC are expected in the USA (1). With the increase in obesity and the decrease in physical activity, the incidence of EC is rising and shows a trend in younger women (2). EC is usually classified into two types to determine the risk of metastasis and recurrence (3). Generally, type I endometrioid endometrial carcinomas (EECs) have a good prognosis and account for $80-85 \%$ of the total cases of EC. By contrast, type II non-EECs are often associated with a worse outcome $(3,4)$. However, the prognostic value of this classification is unsatisfactory, as approximately $20 \%$ of type I tumors recur, whereas $50 \%$ of type II tumors recur (5). A number of previous studies have demonstrated the utility of molecular alterations as prognostic markers, including p53 (6), phosphatase and tensin homolog (PTEN) (7) and Ki-67 (8); however, their value is limited (9). Thus, a deeper understanding of the molecular mechanisms responsible for EC is required for risk stratification and a clinical decision regarding individualized treatment strategies.

Recent studies have indicated that only $2 \%$ of transcripts are protein-coding RNAs, and up to $90 \%$ of the genomic DNA in eukaryotic genomes produces non-coding RNA (10). Initially, non-coding RNAs were regarded as 'junk'; however, numerous studies have confirmed that non-coding RNAs, including microRNAs and long non-coding RNAs (lncRNAs) participate in diverse biological processes (11). Moreover, lncRNAs are regarded as drivers of tumor suppressive and oncogenic functions in various types of cancer $(12,13)$. For example, growth arrest-specific 5 (GAS5) is a tumor suppressor lncRNA; GAS5 induces growth arrest and apoptosis in several mammalian cell lines, whereas the loss of GAS5 is associated with tumor formation (14). Several studies have indicated that lncRNAs contribute to oncogenesis. Certain lncRNAs, such as $H 19$, metastasis-associated lung adenocarcinoma transcript 1 (MALAT1), homeobox (HOX) transcript antisense intergenic RNA (HOTAIR), steroid receptor RNA activator (SRA) and 
prostate cancer-associated ncRNA transcript-1 (PCAT-1) are overexpressed in a variety of human cancers, suggesting their involvement in tumorigenesis (15-19). However, the current knowledge is only the first step toward understanding the biological role of IncRNAs in cancer. Eventually, new discoveries will be used to design novel therapeutic agents (20), particularly for the treatment of patients with recurring EC.

HOTAIR, a well-characterized IncRNA, has gained attention as it has been investigated in numerous solid tumors, including breast (17,21), liver (22), colorectal (23) and lung cancers (24). In these tumors, HOTAIR is upregulated and correlates with invasiveness and metastasis. HOTAIR has been identified as a predictor of overall survival and progressionfree survival in several types of cancer. These studies have also indicated that HOTAIR serves as a modular scaffold. HOTAIR has two important domains, with polycomb repressive complex 2 (PRC2) binding to the 5' end and the complex of lysine-specific demethylase 1 (LSD1) with its co-repressor protein, CoREST1, binding to the $3^{\prime}$ end (25). Thus, HOTAIR can coordinate two complexes to control the state of chromatin. In this way, HOTAIR epigenetically regulates the expression of the HOXD gene cluster, as well as other genes. However, to the best of our knowledge, no previous study has reported the role of HOTAIR in EC.

In the present study, we investigated the expression pattern of HOTAIR in EC and investigated the association between HOTAIR expression and clinicopathological characteristics. Our results demonstrated that HOTAIR was overexpressed in EC and in particular, was highly upregulated in EC that subsequently metastasized and had an unfavorable prognosis. Thus, we evaluated HOTAIR as a possible biomarker for the prognosis of EC.

\section{Materials and methods}

Patients and samples. Sixty-six fresh-frozen samples were obtained from patients pathologically diagnosed with EC who underwent an initial hysterectomy at International Peace Maternity and Child Health Hospital of the China Welfare Institute, affiliated to Shanghai Jiao Tong University School of Medicine (Shanghai, China) between 2008 and 2012. Thirty normal, age-matched endometrial tissues were obtained from patients who underwent hysterectomy due to benign diseases, such as myoma or adenomyosis. Following surgical excision, the tissue specimens were immediately frozen in liquid nitrogen and stored until total RNA extraction. A total of 129 formalin-fixed paraffin-embedded (FFPE) tissues were collected for in situ hybridization (ISH) and immunohistochemical analysis. Apart from the 96 matched frozen cases, the FFPE specimens also included another 21 EC tissues and 12 atypical hyperplasia tissues. The details regarding the patients are presented in Tables I and II. Pathological diagnoses were performed by a gynecologic pathologist according to the classification system of the International Federation of Gynecology and Obstetrics (FIGO). All frozen and FFPE tissues were subjected to hematoxylin and eosin staining to confirm the histological composition. None of the patients enrolled in this study received hormone therapy, radiotherapy, or chemotherapy prior to surgery. This study was approved by the medical Ethics Committee of the International Peace
Maternity and Child Health Hospital. Informed consent was obtained from each patient.

Quantitative reverse transcription PCR ( $q R T-P C R)$. After grinding the tissues, total RNA was extracted using TRIzol reagent (Invitrogen Life Technologies, Carlsbad, CA, USA) according to the manufacturer's instructions. RNA was reverse transcribed into cDNA using the PrimeScript ${ }^{\mathrm{TM}} \mathrm{RT}$ reagent kit with gDNA Eraser (Takara, Dalian, China). Quantitative PCR was employed to detect the relative expression levels of target genes using the SYBR Premix ExTaq II kit (Takara) on the Mastercycler ep realplex (Eppendorf, Hamburg, Germany). Each reaction was performed in triplicate using HOTAIR primers (forward, 5'-GGTAGAAAAAGCAACCACGAAGC-3' and reverse, 5'-ACATAAACCTCTGTCTGTGAGTGCC-3'). The amplification program was as follows: $95^{\circ} \mathrm{C}$ for $30 \mathrm{sec}$, followed by 40 cycles of denaturation at $95^{\circ} \mathrm{C}$ for $5 \mathrm{sec}$ and annealing at $60^{\circ} \mathrm{C}$ for $34 \mathrm{sec}$. GAPDH was used as an internal control. The GAPDH primers were as follows: forward, 5'-CGGATTTGG TCGTATTGGG-3' and reverse, 5'-CTGGAAGATGGTGAT GGGATT-3'. Gene expression was calculated using the $2^{-\Delta \mathrm{Ct}}$ method.

ISH. ISH was employed to analyze HOTAIR expression in the FFPE tissue sections. In brief, the sections were dewaxed, rehydrated and digested with proteinase $\mathrm{K}$. The 5'-digoxigeninlabeled locked nucleic acid-modified HOTAIR probe (Exiqon, Vedbaek, Denmark) was diluted in hybridization solution to $30 \mathrm{nM}$ and incubated with a coverslip at $38^{\circ} \mathrm{C}$ overnight. The following day, after washing, the sections were incubated for $1 \mathrm{~h}$ at $37^{\circ} \mathrm{C}$ with anti-digoxin monoclonal antibody (Boster, Wuhan, China). The sections were then stained with nitro blue tetrazolium/5-bromo-4-chloro-3-indolylpho sphate in the dark. After terminating the reaction, the sections were scored. Positive staining for HOTAIR expression (in blue) was specifically observed in the cytoplasm. The staining scores were evaluated by the intensity and the proportion of HOTAIR-positive cells in 10 random fields under 40 multiple objectives. Positively stained tumor cells were classified as follows: 0 , no positive cells; $1,<10 \% ; 2,10-50 \%$; and 3, $>50 \%$. The staining intensity was scored on a scale of 0 , no staining; 1 , light blue; 2 , blue; and 3 , dark blue. The staining index (SI) was calculated according to the following formula: $\mathrm{SI}=$ staining intensity $\mathrm{x}$ proportion of positively stained cells. Using this method, HOTAIR expression was evaluated using the SI. An SI score of 6 was used as a cut-off value, as previously described (26). The slides were scored independently by two gynecologic pathologists.

Immunohistochemistry. The deparaffinized sections were rehydrated in an ethanol series using a routine method (27). Briefly, the sections were incubated in three washes of xylene for $5 \mathrm{~min}$ each and then incubated in two washes of $75 \%$ ethanol for $10 \mathrm{~min}$ each. This was followed by incubation in two washes of $95 \%$ ethanol for 10 min each and further incubation in two washes of $100 \%$ ethanol for $10 \mathrm{~min}$. Heat-mediated antigen retrieval was then performed in EDTA buffer ( $\mathrm{pH}$ 9.0, Beijing Zhongshan Golden Bridge Biotechnology Co., Ltd., Beijing, China) using microwave treatment for $20 \mathrm{~min}$. Endogenous peroxidase was quenched with $3 \%$ hydrogen peroxide and $10 \%$ goat serum. After the primary antibody was applied, the 
Table I. Expression of HOTAIR in different endometrial tissues.

\begin{tabular}{lcccc}
\hline & & \multicolumn{3}{c}{ HOTAIR in situ hybridization } \\
\cline { 2 - 5 } Group & $\mathrm{n}$ & + & - & Positive rate $(\%)$ \\
\hline Normal endometrium & 30 & 4 & 26 & 13.3 \\
Atypical hyperplasia & 12 & 5 & 7 & 41.7 \\
Endometrial carcinoma & 87 & 63 & 24 & 72.4 \\
\hline
\end{tabular}

Table II. Correlation between HOTAIR expression and clinicopathological parameters in endometrial carcinoma.

\begin{tabular}{|c|c|c|c|c|c|}
\hline \multirow{2}{*}{$\begin{array}{l}\text { Clinicopathological } \\
\text { parameters }\end{array}$} & \multicolumn{5}{|c|}{ HOTAIR in situ hybridization } \\
\hline & $\mathrm{n}$ & + & - & Positive rate $(\%)$ & $\mathrm{p}$-value \\
\hline \multicolumn{6}{|l|}{ Age } \\
\hline$\geq 55$ years & 55 & 40 & 15 & 72.7 & 0.932 \\
\hline$<55$ years & 32 & 23 & 9 & 71.9 & \\
\hline \multicolumn{6}{|l|}{ FIGO stage } \\
\hline I-II & 74 & 51 & 23 & 68.9 & 0.102 \\
\hline III-IV & 13 & 12 & 1 & 92.3 & \\
\hline \multicolumn{6}{|l|}{ Pathological type } \\
\hline Endometrioid & 80 & 58 & 22 & 72.5 & 1.000 \\
\hline Non-endometrioid & 7 & 5 & 2 & 71.4 & \\
\hline \multicolumn{6}{|l|}{ Histological grade } \\
\hline Grade 1 & 43 & 27 & 16 & 62.8 & $0.045^{\mathrm{a}}$ \\
\hline Grade 2 & 27 & 21 & 6 & 77.8 & \\
\hline Grade 3 & 10 & 10 & 0 & 100.0 & \\
\hline \multicolumn{6}{|l|}{ LNM } \\
\hline Positive & 11 & 11 & 0 & 100.0 & $0.031^{\mathrm{a}}$ \\
\hline Negative & 66 & 52 & 24 & 78.9 & \\
\hline \multicolumn{6}{|l|}{ LVS } \\
\hline Positive & 24 & 22 & 2 & 91.7 & $0.015^{\mathrm{a}}$ \\
\hline Negative & 63 & 41 & 22 & 65.1 & \\
\hline \multicolumn{6}{|c|}{ Depth of myometrial invasion } \\
\hline$\leq 1 / 2$ & 69 & 46 & 23 & 66.7 & $0.019^{\mathrm{a}}$ \\
\hline$>1 / 2$ & 18 & 17 & 1 & 94.4 & \\
\hline \multicolumn{6}{|l|}{ ER expression } \\
\hline Positive & 71 & 49 & 22 & 69.0 & 0.216 \\
\hline Negative & 16 & 14 & 2 & 87.5 & \\
\hline \multicolumn{6}{|l|}{ PR expression } \\
\hline Positive & 69 & 47 & 22 & 68.1 & 0.136 \\
\hline Negative & 18 & 16 & 2 & 88.9 & \\
\hline \multicolumn{6}{|l|}{ p53 expression } \\
\hline Positive & 15 & 13 & 2 & 100.0 & 0.219 \\
\hline Negative & 72 & 50 & 22 & 70.7 & \\
\hline
\end{tabular}

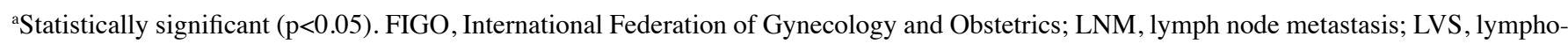
vascular space invasion; ER, estrogen receptor; PR, progesterone receptor.

sections were incubated overnight at $4^{\circ} \mathrm{C}$ in a humidified box. The immunohistochemistry reaction was visualized using a
Histostain-Plus IHC kit (MingRui-Bio Co., Shanghai, China). Primary antibodies used in this study were anti-estrogen 

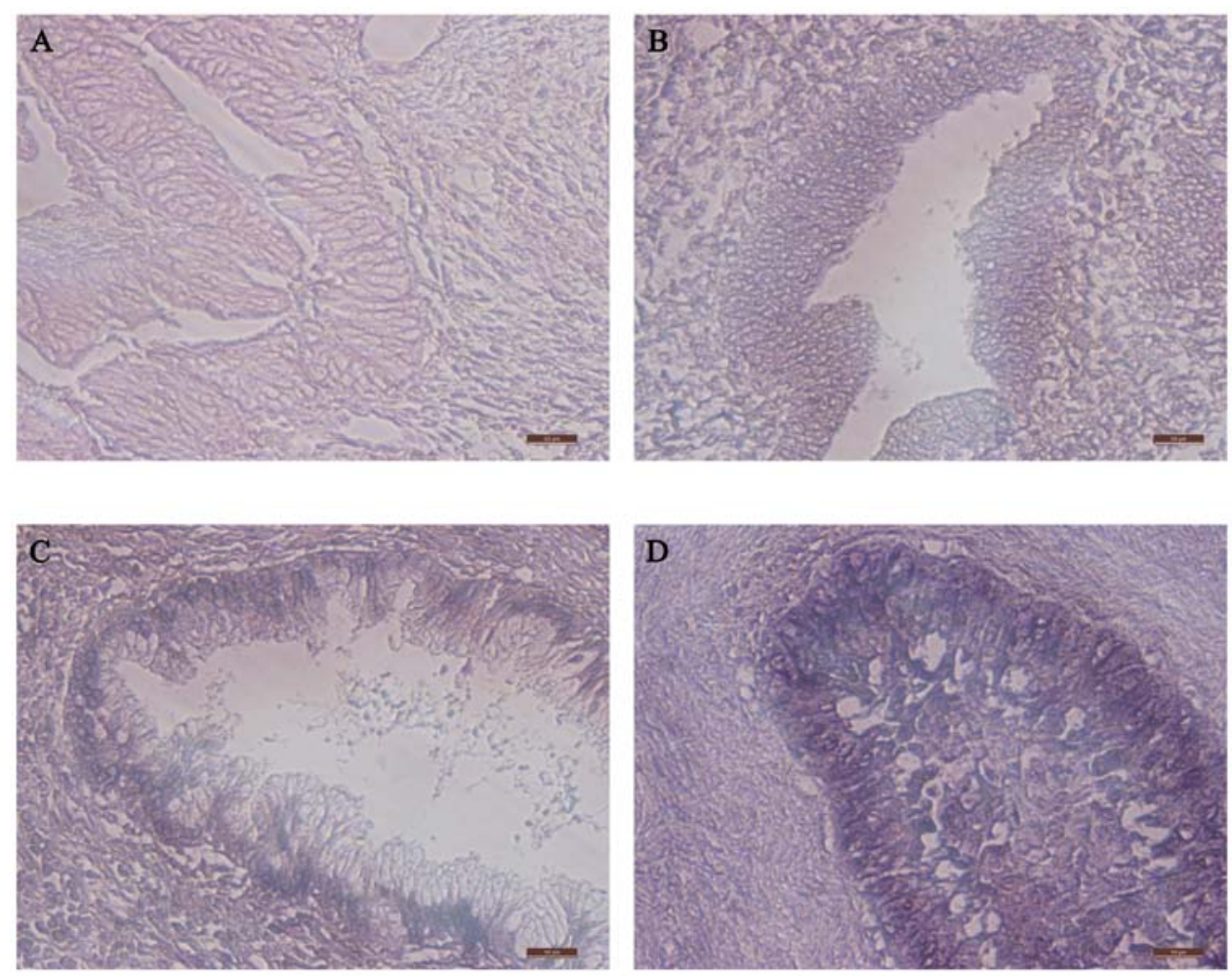

Figure 1. In situ hybridization staining of HOTAIR expression in (A) normal endometrial, (B) atypical, (C) serous endometrial carcinoma and (D) endometrioid carcinoma tissues.

receptor (ER) (diluted 1:100; Cell Signaling Technology, Danvers, MA, USA), anti-progesterone receptor (PR) (diluted 1:100; Cell Signaling Technology) and anti-p53 (diluted 1:150; Cell Signaling Technology). The positively stained tumor cells were classified as follows: no expression, $0-1 \% ; 1, \leq 25 \% ; 2$, $\leq 50 \%$; and $3,>50 \%$. Scores $\geq 2$ were regarded as positive. All slides were visually scored separately scored by two gynecologic pathologists.

lncRNA data mining. An integrated genomic characterization of EC was conducted by The Cancer Genome Atlas (TCGA) network (28). We downloaded Uterine Corpus Endometrial Carcinoma (UCEC) RNA sequencing data from the TCGA website (http://cancergenome.nih.gov/). The clinical information was downloaded from the supplementary file of the TCGA UCEC study (28). The chromosomal locations were obtained from the IncRNA chip annotation file, and the HOTAIR probe termed ASHG19A3A000361 was searched. The relative abundance of IncRNAs was calculated from the raw exon call file using the following formula:

$$
A=\frac{\sum_{i=1}^{n}\left(C_{i} \times L_{i}\right)}{\sum_{j=1}^{m}\left(C_{j} \times L_{j}\right)}
$$

Given that there are $m$ total exons detected in the given sample, $\mathrm{C}_{\mathrm{j}}$ is the read count of the $j$ exon and $L_{j}$ is the length (bp) of this exon. Given that there are $n$ exons detected within the boundary of defined lncRNAs (i.e., exons between txStart and txEnd of the above IncRNAs according to the IncRNA annotation files) and in the same strand, $\mathrm{C}_{\mathrm{i}}$ is the read count of the $i \operatorname{lncRNA}$-exon and $L_{i}$ is the length (bp) of this exon. $A$ is the abundance of the given IncRNA. To ensure the quality, we only used patient data for which at least one raw lncRNA exon read was detected with RNA sequencing. We found 145 patients that expressed HOTAIR from a larger group of patieints with EC with complete clinical data. We then stratified these patients into two groups according to the HOTAIR expression level using the median IncRNA abundance as the threshold (low expression, $\mathrm{n}=83$; high expression, $\mathrm{n}=62$ ).

Statistical analyses. All values are expressed as the means \pm SE. All statistical analyses were performed using the SPSS 17.0 software package (SPSS Inc., Chicago, IL, USA). The differences in HOTAIR expression were evaluated using one-way ANOVA for three-group comparisons and Student's t-tests for two-group comparisons, whereas the associations between HOTAIR expression and clinicopathological characteristics were evaluated with the $\chi^{2}$ test or Fisher's exact test. Survival curves were plotted using the Kaplan-Meier method and compared using the log-rank test. Differences were considered statistically significant at a level of $p<0.05$.

\section{Results}

HOTAIR is overexpressed in EC. To determine the role of HOTAIR in the progression of EC, we first detected HOTAIR expression in EC samples, atypical endometrial hyperplasia tissues and normal endometrial tissues using ISH (Fig. 1). Almost three quarters of the tissue samples from the patients with EC $(63 / 87 ; 72.4 \%)$ showed postive staining for HOTAIR in the cytoplasm of the cancer cells, and almost half of the tissue 
A
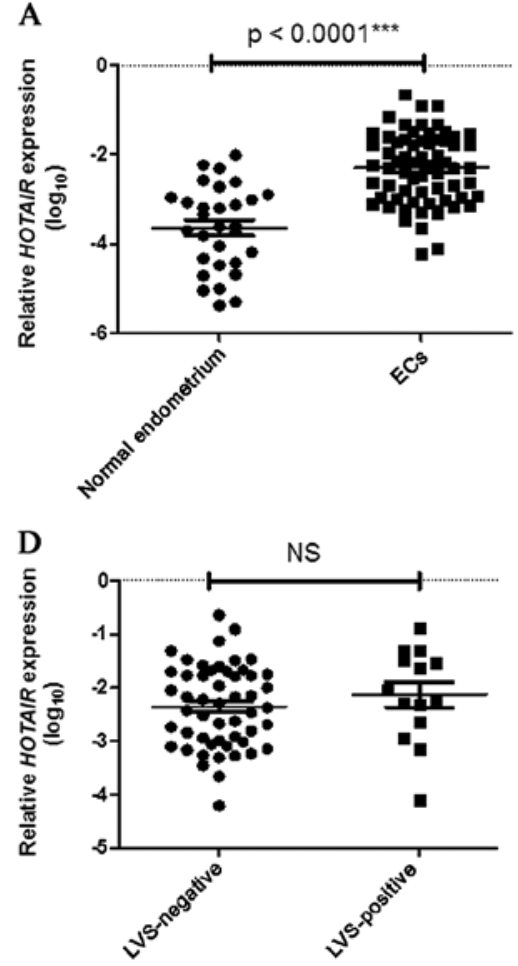

G

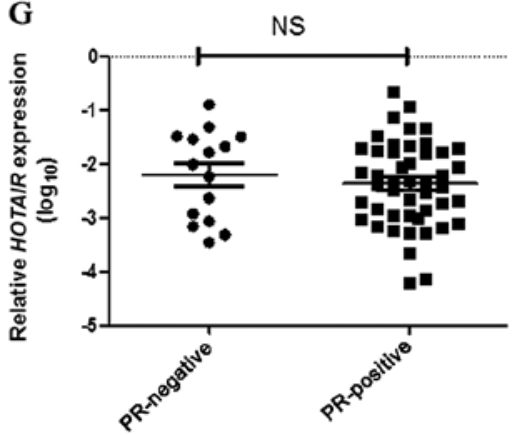

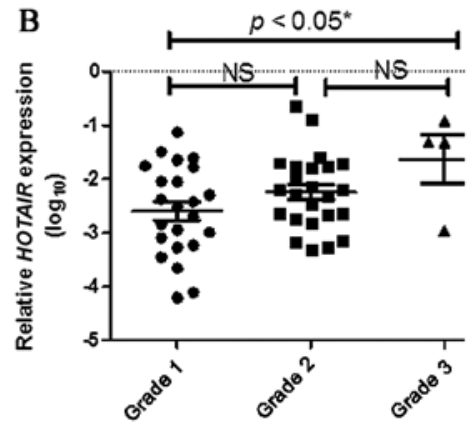

C
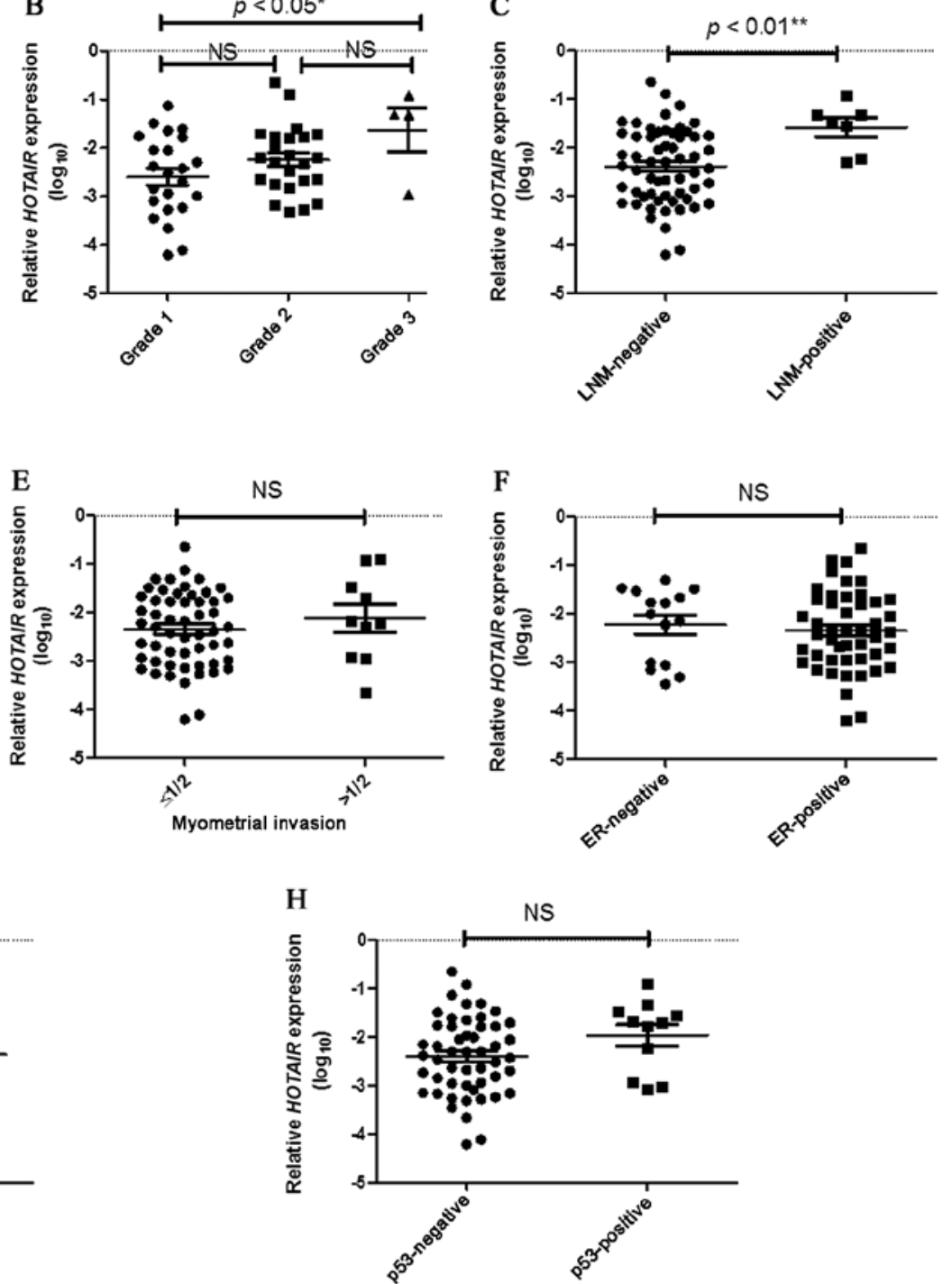

Figure 2. HOTAIR expression and clinicopathological characteristics. The expression of HOTAIR in clinical samples was analyzed using qRT-PCR. HOTAIR expression was compared between (A) normal endometrial and endometrial carcinoma (EC) tissues, (B) histological grade, (C) lymph node metastasis (LNM) status, (D) lymphovascular space invasion (LVS) status, (E) depth of myometrial invasion, (F) estrogen receptor ER expression status, (G) progesterone receptor (PR) expression status and (H) p53 expression status. The relative amount of HOTAIR expressino was calculated using the following equation: $\log _{10}\left[2^{-\Delta C \mathrm{t}}\right]$, where $\Delta \mathrm{CT}=\mathrm{CT}_{\text {HOTAIR }}-\mathrm{CT}_{\mathrm{GAPDH}} \cdot \mathrm{NS}$, not significant.

samples from patients with atypical hyperplasia were shown to express $\operatorname{HOTAIR}(5 / 12 ; 41.7 \%)$; however, the majority of the normal endometrial samples did not show postive staining for HOTAIR (26/30; 86.7\%). HOTAIR expression was increased in the atypical tissues and was even higher in the EC samples ( $\mathrm{p}<0.01$; Table I). In addition, qRT-PCR verified that HOTAIR was significantly upregulated in the majority of the EC tissues, consistent with the results from ISH (Fig. 2).

Correlation between HOTAIR expression and clinicopathological characteristics. To assess the clinical relevance of HOTAIR expression in EC, we examined the correlation between HOTAIR expression in the FFPE tissues as judged by ISH and clinicopathological parameters (Table II). HOTAIR overexpression was markedly associated with tumor grade $(\mathrm{p}=0.045)$, lymph node metastasis $(\mathrm{LNM})(\mathrm{p}=0.031)$, lymphovascular space invasion (LVS) $(\mathrm{p}=0.015)$ and the depth of myometrial invasion $(\mathrm{p}=0.019)$, but not with age, FIGO stage, or pathological type (Table II). ISH also revealed that HOTAIR overexpression was clearly linked to the different degrees of differentiation in the EC tissues (Fig. 3). We further evaluated the correlation between HOTAIR expression levels as judged by qRT-PCR in fresh-frozen tissues and the clinicopathological characteristics (Fig. 2). Our results indicated that HOTAIR expression was higher in grade 3 compared with grade 1 tissues $(\mathrm{p}<0.05)$, and HOTAIR was more highly expressed in the LNM metastasis group $(\mathrm{p}<0.01)$. These data indicate that upregulated HOTAIR expression is associated with metastasis in EC. We also examined the correlation between HOTAIR expression and immunohistochemical parameters. The immunohistochemical staining revealed no correlation between HOTAIR and the expression status of ER ( $\mathrm{p}=0.216)$, PR ( $\mathrm{p}=0.136)$, or p53 ( $\mathrm{p}=0.219)$ (Table II).

Association of HOTAIR with overall survival of patients with $E C$. After downloading the UCEC RNA sequence data from 

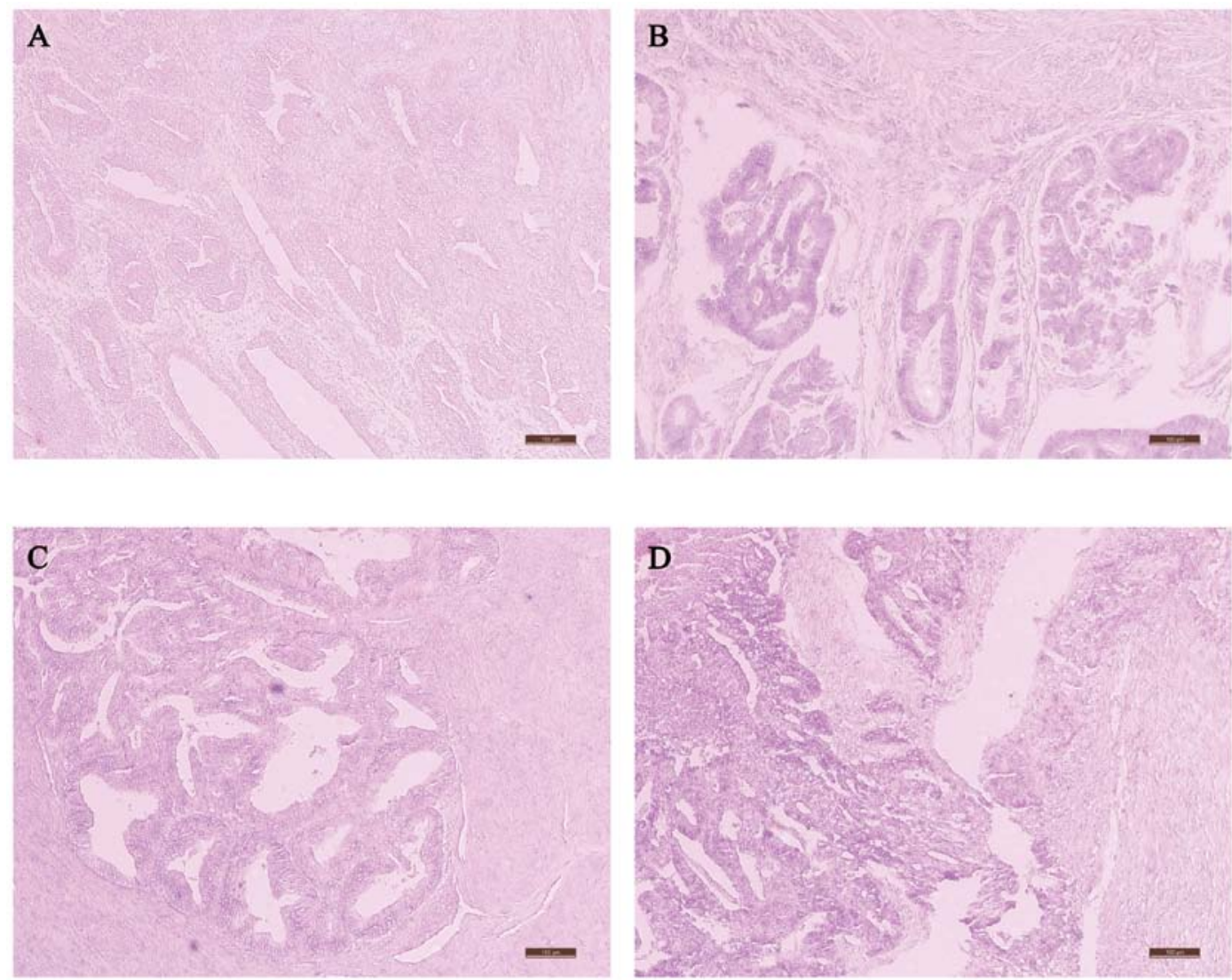

Figure 3. Representative images of HOTAIR expression determined by in situ hybridization of paraffin-embedded tissues with varying degrees of differentiation. (A) Normal endometrial, (B) grade 1, (C) grade 2 and (D) grade 3 tissue.

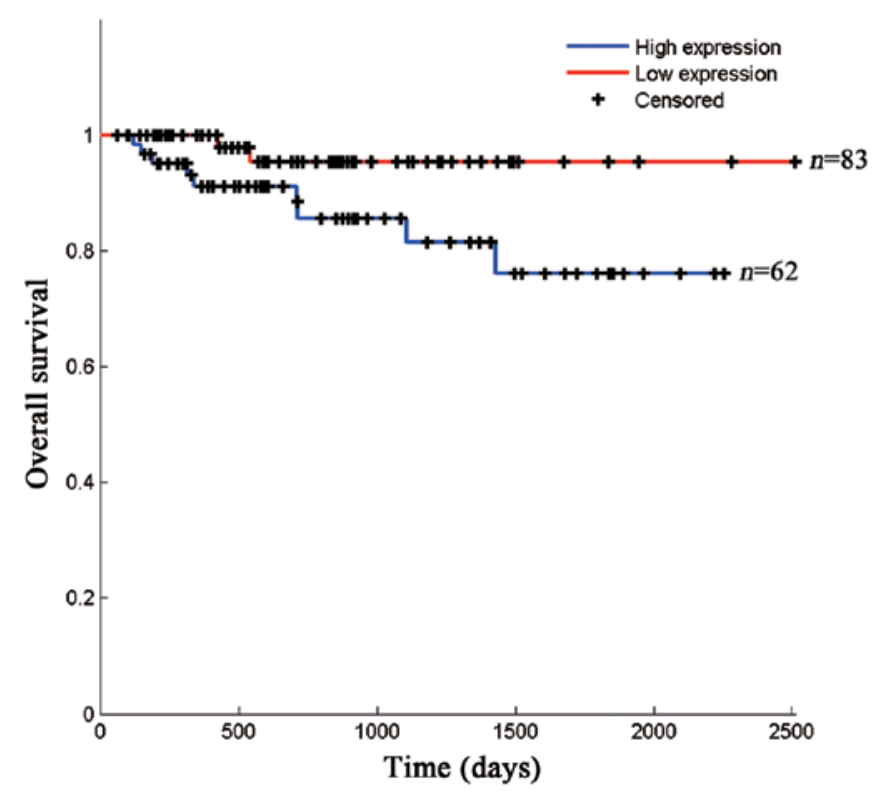

Figure 4. HOTAIR levels are associated with the prognosis of endometrial carcinoma (EC). The Kaplan-Meier curves show that high HOTAIR expression levels correlated with poor survival in the 145 patients (data obtained from TCGA; 141 endometrioid endometrial carcinomas and 4 mixed serous and endometrioid carcinomas). $\mathrm{p}=0.034$.

the TCGA dataset, we calculated the relative expression abundance of HOTAIR. The data of patients from TCGA $(\mathrm{n}=145)$ were stratified according to the median HOTAIR abundance into a low expression group $(\mathrm{n}=83)$ and a high expression group $(n=62)$. These patients included 141 endometrioid endometrial adenocarcinomas and 4 mixed serous and endometrioid carcinomas. The overall survival curves in the low and high HOTAIR mRNA expression groups are shown in Fig. 4. The overall survival was significantly lower in patients with a high HOTAIR expression compared with the patients with a low expression $(\mathrm{p}<0.05)$.

\section{Discussion}

Although the majority of EC cases are treated at an early stage, the disease is still associated with a significantly high mortality rate (29). Thus, further investigation of the pathogenesis of the disease and identification of meaningful prognostic markers is critical. In this study, we demonstrated that HOTAIR was more highly expressed in EC tissues than in normal tissues. Moreover, a higher HOTAIR expression in EC tissues was associated with metastasis and shorter overall survival. Of note, the level of HOTAIR expression estimated using ISH significantly correlated with myometrial invasion and LVS, whereas this correlation was not observed wehn HOTAIR expression as estimated using qRT-PCR. We suspect that the reason for this result may be the number of specimens from the different groups. A previous study suggested that FFPE tissues are a valuable resource for RNA studies, including lncRNA studies, as they can be easily stored and are easily obtained (21). 
Certainly, compared to PCR, the accuracy of the ISH data is poor. Although the significance of the above data is limited by the shortage of PCR samples and the qualitative accuracy of ISH, we confirmed the trend of a high HOTAIR expression in patients with high-grade EC and LNM-positive patients.

To explore the prognostic value of HOTAIR in EC, the availability of a large multi-center population and long-term follow-up is essential. Existing company and university databases that are currently not being investigated should be explored. Although the lncRNA data profile is large, the mining approach has been demonstrated to be effective when using a proper algorithm (30). In addition, when using data mining for medical research, the dataset should be large and confirmed as reliable. Applying these considerations, we performed HOTAIR profiling of data from a large cohort of EC patients obtained from TCGA (28). These data were strong and welldocumented. By analyzing the associations between HOTAIR expression and clinical outcomes of pateints with EC, we found that a high HOTAIR expression was significantly associated with a reduced overall survival. To the best of our knowledge, this is the first report that correlates HOTAIR expression with patient prognosis in EC. Furthermore, the current study also demonstrates that new results from data mining may prove useful in medical research and clinical practice.

Recent studies have suggested that IncRNAs play a major role in oncogenesis. HOTAIR, which is transcribed from the HOX genes, is upregulated in breast cancer (17), hepatocellular carcinoma (22), colorectal cancer (23), pancreatic cancer (31), laryngeal squamous cell carcinoma (32) and gastrointestinal stromal tumors (33). Furthermore, a higher HOTAIR expression has been shown to negatively regulate metastasis-related suppressor genes and promote tumor malignancy (17). By contrast, the depletion of HOTAIR has been shown to reduce the metastatic capacity of tumor cells in vitro (22). A previous study demonstrated that HOTAIR is also linked to chemotherapeutic sensitivity (22). These authors found that silencing HOTAIR expression in HepG2 cells contributes to apoptosis through the tumor necrosis factor- $\alpha$ pathway. Furthermore, they revealed that the knock down of HOTAIR expression sensitizes HepG2 cells to cisplatin and doxorubicin (22). These novel findings clearly indicate that HOTAIR is an important component of tumorigenesis and that the overexpression of HOTAIR is an independent predictor of metastasis and progression-free survival. Our data are consistent with those of previous studies, suggesting that HOTAIR promotes progression in various types of cancer.

HOTAIR expression is transcriptionally induced by estradiol (E2) and is potentially regulated by ERs in breast cancer (34). Bhan et al (34) found that the HOTAIR promoter contains multiple estrogen response elements. Following treatment with E2, HOTAIR transcription is activated, and this activation is coordinated by ERs and several ER co-regulators. A recent study by Chisholm et al (21) demonstrated that HOTAIR is associated with the ER status in breast cancer. However, in our study, significant correlations were not observed between HOTAIR and the ER status. Lu et al (35) detected HOTAIR expression using qRT-PCR in 336 breast cancer patients and found no significant associations with the ER status. Indeed, ER-negative tumors appear to have higher HOTAIR levels, although the difference is not statistically significant $(\mathrm{p}=0.11)(35)$. In our study, the level of HOTAIR expression was significantly higher in ER-negative AN3CA cells than in ER-positive endometrial cells (data not shown). Thus, future studies are required to investigate the mechanisms of action of HOTAIR in EC cells and to determine whether hormones are involved in the expression of HOTAIR.

Previous studies $(17,25)$ have demonstrated that HOTAIR promotes chromatin reprogramming and affects the expression of various target genes, including those involved in apoptosis, tumorigenesis and cell differentiation. In addition, the overexpression of HOTAIR is strongly associated with metastasis and unfavorable prognosis in multiple types of cancer. However, the relevance between the biochemical changes and biological behavior, including invasion and metastasis remains obscure. The processes of metastasis include cancer cell proliferation, local invasion, intravasation, extravasation and colonization. Metastasis is strongly associated with epithelial-to-mesenchymal transition, interaction with inflammatory cells and angiogenesis (36). However, whether HOTAIR is involved in the various processes of metastasis and its exact biological role remain unclear.

In conclusion, we found that HOTAIR was frequently upregulated in EC and was associated with poor differentiation and LNM. The data from 145 patients with EC were mined, and we demonstrated that HOTAIR may be a valuable prognostic molecular marker for EC. However, further studies are required to uncover the underlying mechanisms through which HOTAIR governs EC progression and metastasis. HOTAIR is a potential target for the development of novel therapies for EC.

\section{Acknowledgements}

This study was supported by grants from the National Natural Science Foundation of China (nos. 81072139 and 81172476), the Science and Technology Commission of Shanghai Municipality (no. 12ZR1451400), and the Young Scientific Research Project of Shanghai Municipal Health Bureau (no. 20124Y045).

\section{References}

1. Siegel R, Naishadham D and Jemal A: Cancer statistics, 2013. CA Cancer J Clin 63: 11-30, 2013.

2. Schouten LJ, Goldbohm RA and van den Brandt PA: Anthropometry, physical activity, and endometrial cancer risk: results from the Netherlands Cohort Study. J Natl Cancer Inst 96: 1635-1638, 2004

3. Bokhman JV: Two pathogenetic types of endometrial carcinoma. Gynecol Oncol 15: 10-17, 1983.

4. Boll D, Verhoeven RH, van der Aa MA, et al: Incidence and survival trends of uncommon corpus uteri malignancies in the Netherlands, 1989-2008. Int J Gynecol Cancer 22: 599-606, 2012.

5. Rose PG: Endometrial carcinoma. N Engl J Med 335: 640-649, 1996.

6. Lax SF: Molecular genetic pathways in various types of endometrial carcinoma: from a phenotypical to a molecular-based classification. Virchows Arch 444: 213-223, 2004.

7. Saal LH, Johansson $\mathrm{P}$, Holm K, et al: Poor prognosis in carcinoma is associated with a gene expression signature of aberrant PTEN tumor suppressor pathway activity. Proc Natl Acad Sci USA 104: 7564-7569, 2007

8. Park JY, Kim KR and Nam JH: Immunohistochemical analysis for therapeutic targets and prognostic markers in low-grade endometrial stromal sarcoma. Int J Gynecol Cancer 23: 81-89, 2013. 
9. Salvesen HB, Carter SL, Mannelqvist M, et al: Integrated genomic profiling of endometrial carcinoma associates aggressive tumors with indicators of PI3 kinase activation. Proc Natl Acad Sci USA 106: 4834-4839, 2009.

10. Knauss JL and Sun T: Regulatory mechanisms of long noncoding RNAs in vertebrate central nervous system development and function. Neuroscience 235: 200-214, 2013.

11. Mercer TR, Dinger ME and Mattick JS: Long non-coding RNAs: insights into functions. Nat Rev Genet 10: 155-159, 2009.

12. Gibb EA, Brown CJ and Lam WL: The functional role of long non-coding RNA in human carcinomas. Mol Cancer 10: 38, 2011.

13. Huarte $M$ and Rinn JL: Large non-coding RNAs: missing links in cancer? Hum Mol Genet 19: R152-R161, 2010.

14. Mourtada-Maarabouni M, Pickard MR, Hedge VL, Farzaneh F and Williams GT: GAS5, a non-protein-coding RNA, controls apoptosis and is downregulated in breast cancer. Oncogene 28: 195-208, 2009.

15. Matouk IJ, DeGroot N, Mezan S, et al: The H19 non-coding RNA is essential for human tumor growth. PLoS One 2: e845, 2007.

16. Ji P, Diederichs S, Wang W, et al: MALAT-1, a novel noncoding RNA, and thymosin beta4 predict metastasis and survival in early-stage non-small cell lung cancer. Oncogene 22: 8031-8041, 2003.

17. Gupta RA, Shah N, Wang KC, et al: Long non-coding RNA HOTAIR reprograms chromatin state to promote cancer metastasis. Nature 464: 1071-1076, 2010.

18. Cooper C, Guo J, Yan Y, et al: Increasing the relative expression of endogenous non-coding Steroid Receptor RNA Activator (SRA) in human breast cancer cells using modified oligonucleotides. Nucleic Acids Res 37: 4518-4531, 2009.

19. Ge X, Chen Y, Liao X, et al: Overexpression of long noncoding RNA PCAT-1 is a novel biomarker of poor prognosis in patients with colorectal cancer. Med Oncol 30: 588, 2013.

20. Schalken JA, Hessels D and Verhaegh G: New targets for therapy in prostate cancer: differential display code 3 (DD3(PCA3)), a highly prostate cancer-specific gene. Urology 62: 34-43, 2003.

21. Chisholm KM, Wan Y, Li R, Montgomery KD, Chang HY and West RB: Detection of long non-coding RNA in archival tissue: correlation with polycomb protein expression in primary and metastatic breast carcinoma. PLoS One 7: e47998, 2012.

22. Yang Z, Zhou L, Wu LM, et al: Overexpression of long non-coding RNA HOTAIR predicts tumor recurrence in hepatocellular carcinoma patients following liver transplantation. Ann Surg Oncol 18: 1243-1250, 2011.
23. Kogo R, Shimamura T, Mimori K, et al: Long noncoding RNA HOTAIR regulates polycomb-dependent chromatin modification and is associated with poor prognosis in colorectal cancers. Cancer Res 71: 6320-6326, 2011.

24. Zhuang Y, Wang X, Nguyen HT, et al: Induction of long intergenic non-coding RNA HOTAIR in lung cancer cells by type I collagen. J Hematol Oncol 6: 35, 2013.

25. Tsai MC, Manor O, Wan Y, et al: Long noncoding RNA as modular scaffold of histone modification complexes. Science 329: 689-693, 2010.

26. Nie Y, Liu X, Qu S, Song E, Zou H and Gong C: Long noncoding RNA HOTAIR is an independent prognostic marker for nasopharyngeal carcinoma progression and survival. Cancer Sci 104: 458-464, 2013.

27. Coons AH, Creech HJ and Jones RN: Immunological properties of an antibody containing a fluorescent group. Proc Soc Exp Biol Med 47: 200-202, 1941.

28. Cancer Genome Atlas Research Network, Kandoth C, Schultz N, et al: Integrated genomic characterization of endometrial carcinoma. Nature 497: 67-73, 2013.

29. Amant F, Moerman P, Neven P, Timmerman D, Van Limbergen E and Vergote I: Endometrial cancer. Lancet 366: 491-505, 2005.

30. Zhang XQ, Sun S, Lam KF, et al: A long non-coding RNA signature in glioblastoma multiforme predicts survival. Neurobiol Dis 58: 123-131, 2013.

31. Kim K, Jutooru I, Chadalapaka G, et al: HOTAIR is a negative prognostic factor and exhibits pro-oncogenic activity in pancreatic cancer. Oncogene 32: 1616-1625, 2013.

32. Lv XB, Lian GY, Wang HR, Song E, Yao H and Wang MH: Long noncoding RNA HOTAIR is a prognostic marker for esophageal squamous cell carcinoma progression and survival. PLoS One 8: e63516, 2013

33. Niinuma T, Suzuki H, Nojima M, et al: Upregulation of miR-196a and HOTAIR drive malignant character in gastrointestinal stromal tumors. Cancer Res 72: 1126-1136, 2012

34. Bhan A, Hussain I, Ansari KI, Kasiri S, Bashyal A and Mandal SS Antisense transcript long noncoding RNA (lncRNA) HOTAIR is transcriptionally induced by estradiol. J Mol Biol 425: 3707-3722, 2013.

35. Lu L, Zhu G, Zhang C, et al: Association of large noncoding RNA HOTAIR expression and its downstream intergenic CpG island methylation with survival in breast cancer. Breast Cancer Res Treat 136: 875-883, 2012.

36. Gutschner T and Diederichs S: The hallmarks of cancer: a long non-coding RNA point of view. RNA Biol 9: 703-719, 2012. 